Araştırma Makalesi / Research Article Matematik / Mathematics

Iğdır Üniversitesi Fen Bilimleri Enstitüsü Dergisi, 9(3): 1646-1656, 2019 Journal of the Institute of Science and Technology, 9(3): 1646-1656, 2019

\title{
The Properties of the Altered Pell and Pell Lucas Sequences
}

\section{Fikri KOKEN $^{1 *}$}

\begin{abstract}
The altered Pell and Pell Lucas sequences are defined by altering the Pell and Pell Lucas numbers, it is seen that they have similar properties to usual the Pell and Pell Lucas sequences. Thus, we study some recursive properties of the altered sequences. Further, the greatest common divisors (i.e. GCD) sequences of the altered sequences are investigated, and it is seen that the GCD sequences are subsequences of the Pell and Pell Lucas sequences. Therefore, we obtain Binet formula, the Cassini, Catalan and D'ocagne's identities of the GCD sequences.
\end{abstract}

Keywords: Altered Pell and Pell Lucas numbers, GCD Sequences, Recurrence relations

\section{Değiştirilmiş Pell ve Pell Lucas Dizilerinin Özellikleri}

ÖZET: Değiştirilmiş Pell ve Pell Lucas dizileri, Pell ve Pell Lucas sayıları değiştirilerek tanımlanır, bu dizilerin, Pell ve Pell Lucas dizileriyle benzer özelliklere sahip oldukları görülmektedir. Bu nedenle, değiştirilmiş dizilerin bazı indirgeme özelliklerini incelenir. Ayrıca, değiştirilmiş dizilerin en büyük ortak bölenleri (yani, EBOB) dizileri araştırılır ve EBOB dizilerinin, Pell ve Pell Lucas dizilerinin alt dizileri olduğu görülür. $\mathrm{Bu}$ nedenle, GCD dizilerinin Binet formülünü, Cassini, Catalan ve Docagne'nin eşitlikleri elde edilir.

Anahtar Kelimeler: Değiştirilmiş Pell ve Pell Lucas sayıları, EBOB Dizileri, İndirgeme bağıntıları

\footnotetext{
${ }^{1}$ Fikri KOKEN (Orcid ID: 0000-0002-8304-9525), Eregli Kemal Akman Vocational School, Necmettin Erbakan University, 42310 Konya, Turkey

*Sorumlu Yazar / Corresponding Author: Fikri KOKEN, e-mail: kokenfikri@gmail.com, fkoken@erbakan.edu.tr 


\section{INTRODUCTION}

The Pell $\left\{P_{n}\right\}_{n \geq 0}$ and Pell Lucas $\left\{Q_{n}\right\}_{n \geq 0}$ sequences are established by using same recurrence formulas for the numbers $P_{n}$ and $Q_{n}$ such that $P_{n}=2 P_{n-1}+P_{n-2}$, and $Q_{n}=2 Q_{n-1}+Q_{n-2}, n \geq 2$ with initial conditions $P_{0}=0, P_{1}=1, Q_{0}=2$ and $Q_{1}=2$. The Pell and Pell Lucas numbers are defined by the Binet formulas

$$
P_{n}=\frac{\phi^{n}-\varphi^{n}}{\phi-\varphi} \text { and } Q_{n}=\phi^{n}+\varphi^{n}, \text { for } \phi=1+\sqrt{2}, \varphi=1-\sqrt{2}, n \in \mathbb{N} \text {. }
$$

The terms with negative subscripts are given by $P_{-n}=(-1)^{n-1} P_{n}$ and $Q_{-n}=(-1)^{n} Q_{n}, \quad n \geq 1$ (Horadam,1971). Also, it is known that there is a sequence $\left\{q_{n}\right\}_{n \geq 0}$ holding identities $Q_{n}=2 q_{n}$ and $q_{n}=P_{n}+P_{n-1}$, the numbers $q_{n}$ can be defined by using same recurrence formulas with the Pell and Pell Lucas numbers for $q_{0}=q_{1}=1$ (Horadam,1994; Koshy,2014). In the literature we can reach, the studies of a lot of researchers give the applications of the Pell and Pell Lucas sequences to polynomials and sequences such as the Pell and Pell Lucas polynomials (Horadam and Mahon,1985,1986), their related sequences (Bicknell,1975), the modified Pell numbers (Horadam,1994), some Gaussian Pell and Pell Lucas Numbers (Halıc1 and Oz,2016), the Gaussian Pell polynomials (Halıcı and Öz,2018), the $k$ Pell Quaternions and the $k$ Pell Lucas Quaternions (Gül,2018).

Fibonacci $F_{n}$ and Lucas $L_{n}$ sequences, ones of the most important members of integer sequences, are also associated with a lot of polynomials (Koshy,2001) and some sequences.(McDaniel,1991; Chen,2011, Tasyurdu et al.,2016; Karakas,2017; Gül,2018).

In (Dudley and Tucker,1971), the authors mention from the altered Fibonacci numbers defined as $G_{n}=F_{n}+(-1)^{n}$ and $H_{n}=F_{n}-(-1)^{n}$, these numbers, are not relatively prime for the theirs successive terms, their GCD sequences give some subsequences of the Fibonacci and Lucas sequences (Koshy,2001; Chen,2011). But, GCD sequences of the numbers $L_{n}+(-1)^{n}$ and $L_{n}-(-1)^{n}$ not create important results in a similar way. Thus, different methods are needed for different integer sequences. In (Koken and Arslan,2018), the authors define the altered Pell and Pell Lucas sequences, and also, give gcd properties of these sequences.

The aim of this study is to prove recursive formulas and some identities satisfied by the altered Pell and Pell Lucas sequences. In addition, some GCD sequences of these sequences are established, and obtained basic properties of these GCD sequences such as the Binet formula, Cassini, Catalan and D’ocagne' identities.

\section{MATERIALS AND METHODS}

In this study, we use not only the definitions of the altered Pell sequences $\left\{E_{n}\right\}$ and $\left\{K_{n}\right\}$, which are obtained by altering the Pell sequence, are defined by

$$
E_{n}=\left\{\begin{array}{l}
P_{n}+2, \text { if } n \text { is even } \\
P_{n}-1, \text { otherwise }
\end{array}, \text { and } K_{n}=\left\{\begin{array}{l}
P_{n}-2, \text { if } n \text { is even } \\
P_{n}+1, \text { otherwise }
\end{array},\right.\right.
$$

but also, the altered Pell Lucas sequences $\left\{E_{n}^{\prime}\right\}$ and $\left\{K_{n}^{\prime}\right\}$, which are obtained by altering the Pell Lucas sequence, are defined by 


$$
E_{n}^{\prime}=\left\{\begin{array}{l}
Q_{n}+6, \text { if } n \text { is even } \\
Q_{n}-2, \text { otherwise }
\end{array}, \text { and } K_{n}^{\prime}=\left\{\begin{array}{l}
Q_{n}-6, \text { if } \text { nis even } \\
Q_{n}+2, \text { otherwise }
\end{array} .\right.\right.
$$

In addition, the following Lemma 1 and Lemma 2 are used.

Lemma 1. For the altered Pell numbers, the following identities are valid;

$$
\begin{aligned}
& E_{4 k}=P_{2 k-1} Q_{2 k+1}, E_{4 k+1}=P_{2 k} Q_{2 k+1}, E_{4 k+2}=P_{2 k+2} Q_{2 k}, E_{4 k+3}=P_{2 k+2} Q_{2 k+1}, \\
& K_{4 k}=P_{2 k+1} Q_{2 k-1}, K_{4 k+1}=P_{2 k+1} Q_{2 k}, K_{4 k+2}=P_{2 k} Q_{2 k+2}, K_{4 k+3}=P_{2 k+1} Q_{2 k+2} .
\end{aligned}
$$

Proof From the identities of the Theorem 2 given in (Koken and Arslan, 2018), they are produced.

Lemma 2. For the altered Pell Lucas numbers, the following identities exist;

$$
\begin{aligned}
& E_{4 k}^{\prime}=8 P_{2 k-1} P_{2 k+1}, E_{4 k+1}^{\prime}=8 P_{2 k} P_{2 k+1}, E_{4 k+2}^{\prime}=Q_{2 k+2} Q_{2 k}, E_{4 k+3}^{\prime}=Q_{2 k+2} Q_{2 k+1}, \\
& K_{4 k}^{\prime}=Q_{2 k+1} Q_{2 k-1}, K_{4 k+1}^{\prime}=Q_{2 k+1} Q_{2 k}, K_{4 k+2}^{\prime}=8 P_{2 k} P_{2 k+2}, K_{4 k+3}^{\prime}=8 P_{2 k+1} P_{2 k+2} .
\end{aligned}
$$

Proof From the identities of the Lemma 6 given in (Koken and Arslan, 2018), they are produced.

\section{RESULTS AND DISCUSSION}

As alike it mentioned by authors in (Dudley and Tucker,1971), the Lucas numbers can been not altered with $\{ \pm 1\}$ such as $L_{n}+(-1)^{n}$ and $L_{n}-(-1)^{n}$, then different methods are needed for different integer sequences. Thus, altered Pell and Pell Lucas sequences are dependent on two integer parameters, and also, their GCD sequences are studied with $k$ consecutive terms. This section is occurred two subsections for the altered Pell and altered Pell Lucas numbers.

\section{Some Identities Of The Altered Pell $E_{n}$ And $K_{n}$ Numbers}

In this section, some identities involving the recursive sums of the numbers $E_{n}$ and $K_{n}$ are obtained by the properties of the Pell and Pell numbers. Also, some results concerning the numbers $P_{n, k}^{+}=\operatorname{gcd}\left(E_{n}, E_{n+k}\right)$ and $P_{n, k}^{-}=\operatorname{gcd}\left(K_{n}, K_{n+k}\right)$ defined from greatest common divisors (GCD) for the altered Pell numbers, $E_{n}$ and $K_{n}$, are established. The numbers $P_{n, k}^{+}$and $P_{n, k}^{-}$are called $k$ consecutive GCD numbers of the altered Pell numbers.

Now, several sum relations of consecutive terms of the altered Pell sequences $\left\{E_{n}\right\}$ and $\left\{K_{n}\right\}$ are given;

Theorem 1 Let $E_{n}$ and $K_{n}$ be the $n^{\text {th }}$ altered Pell numbers, then

$$
\begin{gathered}
E_{2 k}+E_{2 k+1}=\left\{\begin{array}{l}
4 P_{k} P_{k+1}, \text { if } k \text { is odd } \\
2 q_{k} q_{k+1}, \text { otherwise }
\end{array}, E_{2 k+1}+E_{2 k+2}=\left\{\begin{array}{l}
2 q_{k+1}^{2}, \text { if } k \text { is odd } \\
4 P_{k+1}^{2}, \text { otherwise }
\end{array},\right.\right. \\
K_{2 k}+K_{2 k+1}=\left\{\begin{array}{l}
2 q_{k} q_{k+1}, \text { if } k \text { is odd } \\
4 P_{k} P_{k+1}, \text { otherwise }
\end{array}, K_{2 k+1}+K_{2 k+2}=\left\{\begin{array}{l}
4 P_{k+1}^{2}, \text { if } k \text { is odd } \\
2 q_{k+1}^{2}, \text { otherwise }
\end{array} .\right.\right.
\end{gathered}
$$

Proof From definition of the number $E_{n}$ by using the property $P_{2 n}=P_{n} Q_{n}$ and substituting $m=k+1$, $n=k$ or $m=k, n=k+1$ in $P_{m+n}=P_{m} Q_{n}-(-1)^{n} P_{m-n}$ for case even or odd $n$, we have 


$$
E_{2 k}+E_{2 k+1}=\left\{\begin{array}{l}
P_{k}\left(Q_{k}+Q_{k+1}\right), \text { if } k \text { is odd } \\
Q_{k}\left(P_{k}+P_{k+1}\right), \text { otherwise }
\end{array} .\right.
$$

By using identities $Q_{k}+Q_{k+1}=4 P_{k+1}$ and $P_{k}+P_{k+1}=q_{k+1}$, desired result is obtained. The other results can be produced by using similar identities.

Theorem 2 Let $E_{n}$ and $K_{n}$ be the $n^{\text {th }}$ altered Pell numbers, then

$$
\begin{gathered}
2 E_{2 k+1}+E_{2 k}=P_{2 k+2}, 2 K_{2 k+1}+K_{2 k}=P_{2 k+2}, \\
2 E_{2 k+2}+E_{2 k+1}=P_{2 k+3}+3,2 K_{2 k+2}+K_{2 k+1}=P_{2 k+3}-3 .
\end{gathered}
$$

Proof From the definitions of the numbers $E_{n}$ and $K_{n}$, by using the recurrence relation of the Pell numbers, all of the desired results are achieved.

The consecutive terms of the altered Pell sequences $\left\{E_{n}\right\}$ and $\left\{K_{n}\right\}$ provide several recurrence relations which are similar to the Fibonacci and Pell sequences, in the following forms, respectively:

$$
\begin{gathered}
2 E_{n+1}+E_{n}=\left\{\begin{array}{l}
E_{n+2}+4, \text { if } n \text { is odd } \\
E_{n+2}-2, \text { otherwise }
\end{array}, 2 K_{n+1}+K_{n}=\left\{\begin{array}{l}
K_{n+2}-4, \text { if } n \text { is odd } \\
K_{n+2}+2, \text { otherwise }
\end{array},\right.\right. \\
E_{n}+2 K_{n+1}=\left\{\begin{array}{l}
K_{n+2}-6, \text { if } n \text { is odd } \\
K_{n+2}+6, \text { otherwise }
\end{array}, 2 E_{n+1}+K_{n}=\left\{\begin{array}{l}
E_{n+2}+6, \text { if } n \text { is odd } \\
E_{n+2}-6, \text { otherwise }
\end{array}\right.\right. \\
E_{n}+E_{n+1}=q_{n+1}+1, K_{n}+K_{n+1}=q_{n+1}-1,
\end{gathered}
$$

and also, any sequences represented as a difference combination of the $E_{n}$ and $K_{n}$ are;

$$
E_{n+1}-E_{n}=\left\{\begin{array}{l}
q_{n}+q_{2}, \text { if } n \text { is odd } \\
q_{n}-q_{2}, \text { otherwise }
\end{array}, K_{n+1}-K_{n}=\left\{\begin{array}{l}
q_{n}-q_{2}, \text { if } n \text { is odd } \\
q_{n}+q_{2}, \text { otherwise }
\end{array} .\right.\right.
$$

Generally, different sum and difference cases of the numbers $E_{n}$ and $K_{n}$ are as follows:

$$
E_{n}+K_{n}=2 P_{n}, E_{n}+K_{n+1}=\left\{\begin{array}{l}
q_{n+1}+q_{2}, \text { if } n \text { is odd } \\
q_{n+1}-q_{2}, \text { otherwise }
\end{array}, E_{n+1}+K_{n}=\left\{\begin{array}{l}
q_{n+1}-q_{2}, \text { if } n \text { is odd } \\
q_{n+1}+q_{2}, \text { otherwise }
\end{array} .\right.\right.
$$

In (Koken and Arslan, 2018), the authors defined two sequences $\left\{P_{n, k}^{+}\right\}=\left\{\operatorname{gcd}\left(E_{n}, E_{n+k}\right)\right\}$ and $\left\{P_{n, k}^{-}\right\}=\left\{\operatorname{gcd}\left(K_{n}, K_{n+k}\right)\right\}, n \geq 1$, and establish a number of identities according to some subsequences of them. Some terms of the sequences $\left\{P_{n, 1}^{+}\right\}_{n \geq 1}$ and $\left\{P_{n, 1}^{-}\right\}_{n \geq 1}$ are given in table 1 .

Table 1: Terms of the sequences $\left\{P_{n, 1}^{+}\right\}$and $\left\{P_{n, 1}^{-}\right\}, 1 \leq n \leq 12$

\begin{tabular}{|c|c|c|c|c|c|c|c|c|c|c|c|c|}
\hline$n$ & 1 & 2 & 3 & 4 & 5 & 6 & 7 & 8 & 9 & 10 & 11 & 12 \\
\hline$P_{n, 1}^{+}$ & 4 & $2 P_{2}$ & 2 & $Q_{3}$ & 4 & $2 P_{4}$ & 2 & $Q_{5}$ & 4 & $2 P_{6}$ & 2 & $Q_{7}$ \\
\hline$P_{n, 1}^{-}$ & 2 & $Q_{2}$ & 2 & $2 P_{3}$ & 2 & $Q_{4}$ & 2 & $2 P_{5}$ & 2 & $Q_{6}$ & 2 & $2 P_{7}$ \\
\hline
\end{tabular}


It is firstly seen that the numbers $P_{2 k, 1}^{+}$are the number $2 P_{k+1}$ for $k=1,3,5, \ldots$, and are the numbers $Q_{k+1}$ for $k=2,4,6, \ldots$, i.e., the sequence $\left\{P_{2 k, 1}^{+}\right\}_{k \geq 1}$ is $\left\{2 P_{2}, Q_{3}, 2 P_{4}, \ldots\right\}$. Also, the numbers $P_{2 k+1,1}^{+}$are 2 for $k=1,3,5, \ldots$, and are 4 for $k=2,4,6, \ldots$. The sequence $\left\{P_{2 k+1,1}^{+}\right\}_{k \geq 1}$ is constant-periodic integer sequence alike $\{2,4,2,4, \ldots\}$. Secondly, notice that the sequence $\left\{P_{n, 1}^{-}\right\}_{n \geq 1}=\left\{\operatorname{gcd}\left(K_{n}, K_{n+1}\right)\right\}$ is constant for the odd integer number $n$, i.e. the $P_{n, 1}^{-}=2$. And also, $P_{2 n, 1}^{-}=Q_{n+1}$ for the odd integer number $n$ and $P_{2 n, 1}^{-}=2 P_{n+1}$ for the even integer numbers $n$.

Lemma 3 Let $P_{n, 1}^{+}$and $P_{n, 1}^{-}, n \geq 1$ be the $n^{\text {th }} 1$-consecutive GCD numbers of the altered Pell numbers, then

$$
P_{2 k, 1}^{+}=\left\{\begin{array}{c}
2 P_{k+1}, \text { for odd } k \\
Q_{k+1}, \text { for even } k
\end{array}, P_{2 k+1,1}^{+}=\left\{\begin{array}{l}
2, \text { for odd } k \\
4, \text { for even } k
\end{array}, P_{2 k, 1}^{-}=\left\{\begin{array}{c}
2 P_{k+1}, \text { for even } k \\
Q_{k+1}, \text { for odd } k
\end{array}, P_{2 k-1,1}^{-}=2 .\right.\right.\right.
$$

Proof From the identities of the Theorem 1 and Theorem 4 given in (Koken and Arslan, 2018), they are achieved.

Theorem 3 There are two difference identities $P_{n, 1}^{+}$and $P_{n, 1}^{-}$as follows

$$
P_{2 k+2,1}^{+}-P_{2 k, 1}^{+}=2 P_{2\left\lceil\frac{k}{2}+1\right\rceil-1}, \quad P_{2 k+2,1}^{-}-P_{2 k, 1}^{-}=2 P_{\left\lceil\frac{k+4}{2}\right\rceil+(-1)^{k}},
$$

where $\lceil x\rceil$ is ceiling integer function, gives the least integer less than or equal to $x$.

Proof By using the definition of the $P_{n, 1}^{+}$and the identity $q_{n}=P_{n}+P_{n-1}$, we write

$$
\begin{aligned}
P_{2 k+2,1}^{+}-P_{2 k, 1}^{+} & =\left\{\begin{array}{l}
2\left(q_{k+2}-P_{k+1}\right), \text { if } n \text { is odd } \\
2\left(P_{k+2}-q_{k+1}\right), \text { otherwise }
\end{array}\right. \\
& = \begin{cases}2 P_{k+2}, & \text { if } n \text { is } \text { odd } \\
2 P_{k+1}, & \text { otherwise }\end{cases}
\end{aligned}
$$

The desired results are obtained.

Theorem 4 For the $n^{\text {th }}$ number $P_{n, 1}^{+}$, the Pell type recursive relations are valid:

$$
\begin{aligned}
& 2 P_{2 k+2,1}^{+}-P_{2 k, 1}^{+}=\left\{\begin{array}{ll}
2 P_{k+3}, & \text { if } n \text { is odd } \\
2 q_{k+2}, & \text { otherwise }
\end{array}, \quad P_{2 k+2,1}^{+}-2 P_{2 k, 1}^{+}=\left\{\begin{array}{l}
2 q_{k+1}, \text { if } n \text { is odd } \\
-2 P_{k}, \text { otherwise }
\end{array}\right.\right. \\
& 2 P_{2 k+2,1}^{-}-P_{2 k, 1}^{-}=\left\{\begin{array}{ll}
Q_{k+2}, & \text { if } n \text { is odd } \\
2 P_{k+3}, & \text { otherwise }
\end{array}, \quad P_{2 k+2,1}^{-}-2 P_{2 k, 1}^{-}=\left\{\begin{array}{ll}
-2 P_{k}, & \text { if } n \text { is odd } \\
Q_{k+1}, & \text { otherwise }
\end{array} .\right.\right.
\end{aligned}
$$

Proof By using the definition of the $P_{n, 1}^{+}$, and identities $q_{n}=P_{n}+P_{n-1}$ and $P_{n-1}+2 P_{n}=P_{n+1}$, we write 


$$
\begin{gathered}
2 P_{2 k+2,1}^{+}-P_{2 k, 1}^{+}=\left\{\begin{array}{l}
2\left(2 q_{k+2}-P_{k+1}\right), \text { if } n \text { is odd } \\
2\left(2 P_{k+2}-q_{k+1}\right), \text { otherwise }
\end{array}\right. \\
P_{2 k+2,1}^{+}-2 P_{2 k, 1}^{+}=\left\{\begin{array}{l}
2\left(q_{k+2}-2 P_{k+1}\right), \text { if } n \text { is odd } \\
2\left(P_{k+2}-2 q_{k+1}\right), \text { otherwise }
\end{array}\right.
\end{gathered}
$$

Corollary 1 The numbers $P_{4 k+k_{1}, 1}^{+}$and $P_{4 k+k_{1}, 1}^{-}$are either the Pell and Pell Lucas numbers or constant in the following equations:

$$
\begin{aligned}
& P_{4 k, 1}^{+}=Q_{2 k+1}, P_{4 k+1,1}^{+}=4, P_{4 k+2,1}^{+}=2 P_{2 k+2}, P_{4 k+3,1}^{+}=2, \\
& P_{4 k, 1}^{-}=2 P_{2 k+1}, P_{4 k+1,1}^{-}=2, P_{4 k+2,1}^{-}=Q_{2 k+2}, P_{4 k+3,1}^{-}=2 .
\end{aligned}
$$

Proof From the Lemma 3, when the values of $k$ vary according to odd and even, subscripts of these numbers takes the desired values.

When the values of indices vary according to these numbers in the Corollary 1 , we obtain in the following results:

Corollary 2 There exist some relations between the numbers $P_{n, 1}^{+}$and $P_{n, 1}^{-}$;

$$
\begin{gathered}
P_{4 k+2,1}^{+}-P_{4 k, 1}^{+}=2 P_{2 k+1}, \quad P_{4 k+2,1}^{-}-P_{4 k, 1}^{-}=2 P_{2 k+2}, \quad P_{4 k, 1}^{+}-P_{4 k-2,1}^{+}=2 P_{2 k+1}, \\
P_{4 k, 1}^{-}-P_{4 k-2,1}^{-}=2 P_{2 k}, \quad P_{4 k+2,1}^{+}-P_{4 k-2,1}^{+}=4 P_{2 k+1}, \quad P_{4 k+2,1}^{-}-P_{4 k-2,1}^{-}=2 Q_{2 k+1} . \\
2 P_{4 k+2,1}^{+}-P_{4 k, 1}^{+}=Q_{2 k+2}, \quad 2 P_{4 k+2,1}^{-}-P_{4 k, 1}^{-}=2 P_{2 k+3}, \\
2 P_{4 k, 1}^{+}-P_{4 k-2,1}^{+}=2 P_{2 k+2}, \quad 2 P_{4 k, 1}^{-}-P_{4 k-2,1}^{-}=Q_{2 k+1} .
\end{gathered}
$$

Theorem 5 Some recurrence relation of the number $P_{n, 1}^{+}$and $P_{n, 1}^{-}$are

$$
\begin{aligned}
& P_{4 k+4,1}^{+}=4 P_{4 k+2,1}^{+}-P_{4 k, 1}^{+}, P_{4 k+4,1}^{-}=2 P_{4 k+2,1}^{-}-P_{4 k, 1}^{-}, \\
& P_{4 k+2,1}^{+}=2 P_{4 k, 1}^{+}-P_{4 k-2,1}^{+}, P_{4 k+2,1}^{-}=4 P_{4 k, 1}^{-}-P_{4 k-2,1}^{-} .
\end{aligned}
$$

Proof By using the definition of the $P_{n, 1}^{+}$, and identities $2 P_{n}-q_{n-1}=q_{n}$ and $2 P_{n}+q_{n}=q_{n+1}$, we write

$$
4 P_{4 k+2,1}^{+}-P_{4 k, 1}^{+}=2\left(4 P_{2 k+2}-q_{2 k+1}\right)=2\left(2 P_{2 k+2}+q_{2 k+2}\right)=2 q_{2 k+3} .
$$

And also, we rewrite

$$
2 P_{4 k, 1}^{+}-P_{4 k-2,1}^{+}=2\left(P_{2 k+2}+P_{2 k}\right)=2 Q_{2 k+1} .
$$

The other results are done in a similar way.

Corollary 3 There exist some relations between the numbers $P_{n, 1}^{+}$and $P_{n, 1}^{-}$;

$$
\begin{aligned}
& P_{4 k+4,1}^{+}-P_{4 k, 1}^{+}=2 Q_{2 k+2}=2 P_{4 k+2,1}^{-}, P_{4 k+4,1}^{-}-P_{4 k, 1}^{-}=4 P_{2 k+2}=2 P_{4 k+2,1}^{+}, \\
& P_{4 k+2,1}^{+}-P_{4 k-2,1}^{+}=4 P_{2 k+1}=2 P_{4 k, 1}^{-}, P_{4 k+2,1}^{-}-P_{4 k-2,1}^{-}=2 Q_{2 k+1}=2 P_{4 k, 1}^{+} .
\end{aligned}
$$


Theorem 6 The Binet formulas for the numbers $P_{n, 1}^{+}$and $P_{n, 1}^{-}$are

$$
P_{4 k, 1}^{+}=\phi^{2 k+1}+\varphi^{2 k+1}, P_{4 k+2,1}^{+}=\frac{\phi^{2 k+2}-\varphi^{2 k+2}}{\sqrt{2}}, P_{4 k, 1}^{-}=\frac{\phi^{2 k+1}-\varphi^{2 k+1}}{\sqrt{2}}, P_{4 k+2,1}^{-}=\phi^{2 k+2}+\varphi^{2 k+2} .
$$

Proof By using identities given in (1), we obtain desired results.

For the $P_{n, 1}^{+}$and $P_{n, 1}^{-}$, some well-known identities such as the Cassini, Catalan and D'ocagne's identities can be established;

Theorem 7 For the $P_{4 k, 1}^{+}$and $P_{4 k, 1}^{-}$, Cassini, Catalan and D'ocagne's like identities are

$$
\begin{gathered}
P_{4 k+4,1}^{+} P_{4 k-4,1}^{+}-\left(P_{4 k, 1}^{+}\right)^{2}=-2^{5}, \quad P_{4(k+r), 1}^{+} P_{4(k-r), 1}^{+}-\left(P_{4 k, 1}^{+}\right)^{2}=-2^{3} P_{2 r}^{2}, \\
P_{4 k_{1}+4,1}^{+} P_{4 k_{2}, 1}^{+}-P_{4 k_{1}, 1}^{+} P_{4 k_{2}+4,1}^{+}=2^{2} P_{2\left(k_{2}-k_{1}\right)}, \quad P_{4 k+4,1}^{-} P_{4 k-4,1}^{-}-\left(P_{4 k, 1}^{-}\right)^{2}=2^{4}, \\
P_{4(k+r), 1}^{-} P_{4(k-r), 1}^{-}-\left(P_{4 k, 1}^{-}\right)^{2}=2^{2} P_{2 r}^{2}, \quad P_{4 k_{1}+4,1}^{-} P_{4 k_{2}, 1}^{-}-P_{4 k_{1}, 1}^{-} P_{4 k_{2}+4,1}^{-}=2^{3} P_{2\left(k_{1}-k_{2}\right)} .
\end{gathered}
$$

Theorem 8 Let $P_{n, 2}^{+}$and $P_{n, 2}^{-}, n \geq 1$ be the $n^{\text {th }}$ 2-consecutive GCD numbers of the altered Pell numbers, then

$$
P_{4 k+1,2}^{+}=2 Q_{2 k+1}, P_{4 k+3,2}^{+}=2 P_{2 k+2}, P_{4 k+1,2}^{-}=6 P_{2 k+1}, P_{4 k+3,2}^{-}=Q_{2 k+2} .
$$

Proof By using the Lemma 1 and the GCD properties of the Pell and Pell Lucas numbers, we obtain the desired results.

Lemma 4 Let $P_{n, 3}^{+}$and $P_{n, 3}^{-}, n \geq 1$ be the $n^{\text {th }} 3$-consecutive GCD numbers of the altered Pell numbers, then

$$
P_{4 k, 3}^{+}=\left\{\begin{array}{l}
5 Q_{2 k+1}, k \equiv 2 \bmod (3) \\
Q_{2 k+1}, \quad \text { otherwise }
\end{array}, P_{4 k+2,3}^{-}=\left\{\begin{array}{c}
5 Q_{2 k+2}, k \equiv 0 \bmod (3) \\
Q_{2 k+2}, \quad \text { otherwise }
\end{array}, P_{4 k+2,3}^{+}=2 P_{2 k+2}, P_{4 k, 3}^{-}=2 P_{2 k+1} .\right.\right.
$$

Proof From Theorem 6 given in (Koken and Arslan, 2018), they are achieved.

The $\left\{P_{4 k+k_{1}, r}^{+}\right\}=\left\{\left(E_{4 k+k_{1}}, E_{4 k+k_{1}+r}\right)\right\}$ and $\left\{P_{4 k+k_{1}, r}^{-}\right\}=\left\{\left(K_{4 k+k_{1}}, K_{4 k+k_{1}+r}\right)\right\} \quad r \geq 1, \quad k_{1}=0,1,2,3$ are trivial constant for some integer $r$, for example $\left\{P_{4 k, 2}^{+}\right\}=2$ and $\left\{P_{4 k+2,2}^{-}\right\}=2$, and also a number of different r-consecutive GCD numbers of the altered Pell sequences can be produced. In addition, for sequences given in Theorem 8 and Lemma 4, a number of identities can be given similar to identities of the $P_{n, 1}^{+}$and $P_{n, 1}^{-}$. For instance; Binet formulas of some number are

$$
P_{4 k+1,2}^{+}=2\left(\phi^{2 k+1}+\varphi^{2 k+1}\right), P_{4 k+1,2}^{-}=\frac{3\left(\phi^{2 k+1}-\varphi^{2 k+1}\right)}{\sqrt{2}}, P_{4 k+2,3}^{+}=\frac{\phi^{2 k+2}-\varphi^{2 k+2}}{\sqrt{2}}, P_{4 k, 3}^{-}=\frac{\phi^{2 k+1}-\varphi^{2 k+1}}{\sqrt{2}} .
$$




\section{Some Properties Of The Altered Pell Lucas Numbers $E_{n}^{\prime}$ And $K_{n}^{\prime}$}

In this section, by using the properties satisfied by the Pell and Pell numbers, we obtain some identities involving recurrences sums of the numbers $E_{n}^{\prime}$ and $K_{n}^{\prime}$. Also, some results concerning the numbers $Q_{n, k}^{+}=\operatorname{gcd}\left(E_{n}^{\prime}, E_{n+k}^{\prime}\right)$ and $Q_{n, k}^{-}=\operatorname{gcd}\left(K_{n}^{\prime}, K_{n+k}^{\prime}\right)$ defined from the greatest common divisors for the altered Pell Lucas sequences, $\left\{E_{n}^{\prime}\right\}$ and $\left\{K_{n}^{\prime}\right\}$, are established. Now, some sum relations of consecutive terms of the altered Pell Lucas sequences $\left\{E_{n}^{\prime}\right\}_{n \geq 1}$ and $\left\{K_{n}^{\prime}\right\}_{n \geq 1}$ are given;

Theorem 9 Let $E_{n}^{\prime}$ and $K_{n}^{\prime}$ be the $n^{\text {th }}$ altered Pell Lucas numbers, then

$$
\begin{aligned}
& E_{2 k}^{\prime}+E_{2 k+1}^{\prime}=\left\{\begin{array}{l}
4 P_{k} Q_{k+1}, \text { if } k \text { is odd } \\
4 P_{k+1} Q_{k}, \text { otherwise }
\end{array}, E_{2 k+1}^{\prime}+E_{2 k+2}^{\prime}=\left\{\begin{array}{l}
4\left(Q_{k} P_{k+2}+q_{2}\right), \text { if } k \text { is odd } \\
4\left(Q_{k} P_{k+2}-q_{1}\right), \text { otherwise }
\end{array},\right.\right. \\
& K_{2 k}^{\prime}+K_{2 k+1}^{\prime}=\left\{\begin{array}{l}
4 P_{k+1} Q_{k}, \text { if } k \text { is odd } \\
4 P_{k} Q_{k+1}, \text { otherwise }
\end{array}, K_{2 k+1}^{\prime}+K_{2 k+2}^{\prime}=\left\{\begin{array}{l}
4\left(Q_{k} P_{k+2}+q_{1}\right), \text { if } k \text { is odd } \\
4\left(Q_{k} P_{k+2}-q_{2}\right), \text { otherwise }
\end{array}\right.\right.
\end{aligned}
$$

Proof From the definitions of the numbers $E_{n}^{\prime}$ and $K_{n}^{\prime}$, by using the properties $P_{k}+P_{k+1}=q_{k+1}$, $P_{2 k}=P_{k} Q_{k}, Q_{k}+Q_{k+1}=4 P_{k+1}$ and $Q_{m+n}=Q_{m} Q_{n}-(-1)^{n} Q_{m-n}$ for case even or odd $n$, we have all desired results.

Theorem 10 Let $E_{n}^{\prime}$ and $K_{n}^{\prime}$ be the $n^{\text {th }}$ altered Pell Lucas numbers, then

$$
\begin{array}{cl}
3 E_{2 k+1}^{\prime}+E_{2 k}^{\prime}=4 P_{2 k+2}, 3 K_{2 k+1}^{\prime}+K_{2 k}^{\prime}=4 P_{2 k+2}, \\
2 E_{2 k+2}^{\prime}+E_{2 k+1}^{\prime}=Q_{2 k+3}+10,2 K_{2 k+2}^{\prime}+K_{2 k+1}^{\prime}=Q_{2 k+3}-10 .
\end{array}
$$

Proof From the definitions of the numbers $E_{n}^{\prime}$ and $K_{n}^{\prime}$, by using the recurrence relation of the Pell Lucas numbers and the property $Q_{k}+Q_{k+1}=4 P_{k+1}$, we achieve all results.

The terms of the altered Pell Lucas sequences $\left\{E_{n}^{\prime}\right\}$ and $\left\{K_{n}^{\prime}\right\}$ satisfy several recurrence relations which are similar to equations given for the Lucas and Pell Lucas numbers. The Pell Lucas type recurrence relations are valid:

$$
\begin{aligned}
& 2 E_{n+1}^{\prime}+E_{n}^{\prime}=\left\{\begin{array}{l}
E_{n+2}^{\prime}+12, \text { if } n \text { is odd } \\
E_{n+2}^{\prime}+4, \text { otherwise }
\end{array}, \quad 2 K_{n+1}^{\prime}+K_{n}^{\prime}=\left\{\begin{array}{c}
K_{n+2}^{\prime}-12, \text { if } n \text { is odd } \\
K_{n+2}^{\prime}-4, \text { otherwise }
\end{array},\right.\right. \\
& E_{n}^{\prime}+2 K_{n+1}^{\prime}=\left\{\begin{array}{l}
K_{n+2}^{\prime}-16, \text { if } n \text { is odd } \\
K_{n+2}^{\prime}+16, \text { otherwise }
\end{array}, 2 E_{n+1}^{\prime}+K_{n}^{\prime}=\left\{\begin{array}{l}
E_{n+2}^{\prime}+16, \text { if } n \text { is odd } \\
E_{n+2}^{\prime}-16, \text { otherwise }
\end{array} .\right.\right.
\end{aligned}
$$

The Lucas type relations are $E_{n}^{\prime}+E_{n+1}^{\prime}=4\left(P_{n+1}+1\right)$ and $K_{n}^{\prime}+K_{n+1}^{\prime}=4\left(P_{n+1}-1\right)$. And also, the linear differences of these numbers are

$$
E_{n+1}^{\prime}-E_{n}^{\prime}=\left\{\begin{array}{ll}
4\left(P_{n}+2\right), & \text { if } n \text { is odd } \\
4\left(P_{n}-2\right), & \text { otherwise }
\end{array}, K_{n+1}^{\prime}-K_{n}^{\prime}=\left\{\begin{array}{ll}
4\left(P_{n}-2\right), & \text { if } n \text { is odd } \\
4\left(P_{n}+2\right), & \text { otherwise }
\end{array} .\right.\right.
$$

The following relationships are valid between the numbers $E_{n}^{\prime}$ and $K_{n}^{\prime}$ : 


$$
E_{n}^{\prime}+K_{n}^{\prime}=2 Q_{n}, E_{n}^{\prime}+K_{n+1}^{\prime}=\left\{\begin{array}{l}
4\left(P_{n+1}-2\right), \text { if } n \text { is odd } \\
4\left(P_{n+1}+2\right), \text { otherwise }
\end{array}, E_{n+1}^{\prime}+K_{n}^{\prime}=\left\{\begin{array}{c}
4\left(P_{n+1}+2\right), \text { if } n \text { is odd } \\
4\left(P_{n+1}-2\right), \text { otherwise }
\end{array} .\right.\right.
$$

In (Koken and Arslan, 2018), the authors give the $Q_{n, k}^{+}=\operatorname{gcd}\left(E_{n}^{\prime}, E_{n+k}^{\prime}\right)$ and $Q_{n, k}^{-}=\operatorname{gcd}\left(K_{n}^{\prime}, K_{n+k}^{\prime}\right)$ numbers defined from greatest common divisors for the altered Pell Lucas numbers, a few terms of these sequences are given in table 2 .

Table 2: The terms of the sequences $\left\{E_{n}^{\prime}\right\}$ and $\left\{K_{n}^{\prime}\right\}, 1 \leq n \leq 12$

\begin{tabular}{|c|c|c|c|c|c|c|c|c|c|c|c|c|}
\hline $\mathrm{n}$ & 1 & 2 & 3 & 4 & 5 & 6 & 7 & 8 & 9 & 10 & 11 & 12 \\
\hline$Q_{n, 1}^{+}$ & 12 & $2 Q_{2}$ & 4 & $8 P_{3}$ & 4 & $2 Q_{4}$ & 4 & $8 P_{5}$ & 12 & $2 Q_{6}$ & 4 & $8 P_{7}$ \\
\hline$Q_{n, 1}^{-}$ & 4 & $8 P_{2}$ & 4 & $2 Q_{3}$ & 12 & $8 P_{4}$ & 4 & $2 Q_{5}$ & 4 & $8 P_{6}$ & 4 & $2 Q_{7}$ \\
\hline
\end{tabular}

Lemma 5 If $Q_{n, 1}^{+}, n \geq 1$ is the $n^{\text {th }}$ term of the 1-consecutive GCD sequences of the altered Pell Lucas sequences, then

$$
\begin{gathered}
Q_{2 k, 1}^{+}=\left\{\begin{array}{l}
2 Q_{k+1}, \text { for } \text { odd } k \\
8 P_{k+1}, \text { for } \text { even } k
\end{array}, \quad Q_{2 k-1,1}^{+}=\left\{\begin{array}{l}
12, \text { if } k \equiv 1(\bmod 4) \\
4, \quad \text { otherwise }
\end{array},\right.\right. \\
Q_{4 k, 1}^{+}=8 P_{2 k+1}, Q_{4 k+1,1}^{+}=\left\{\begin{array}{c}
12, \text { if } k \equiv 0,2(\bmod 4) \\
4, \quad \text { otherwise }
\end{array}, Q_{4 k+2,1}^{+}=2 Q_{2 k+2}, Q_{4 k-1,1}^{+}=4 .\right.
\end{gathered}
$$

Proof All results are seen from identities of the Theorem 7 and Theorem 8 given in (Koken and Arslan, 2018).

Lemma 6 If $Q_{n, 1}^{-}, n \geq 1$ is the $n^{\text {th }}$ terms of the 1 -consecutive GCD sequences the altered Pell Lucas sequences, then

$$
\begin{gathered}
Q_{2 k, 1}^{-}=\left\{\begin{array}{l}
8 P_{k+1}, \text { for odd } k \\
2 Q_{k+1}, \text { for even } k
\end{array}, Q_{2 k-1,1}^{-}=\left\{\begin{array}{l}
12, \text { if } k \equiv 3(\bmod 4) \\
4, \quad \text { otherwise }
\end{array},\right.\right. \\
Q_{4 k, 1}^{-}=2 Q_{2 k+1}, Q_{4 k+1,1}^{-}=\left\{\begin{array}{cc}
12, \text { if } k \equiv 1,3(\bmod 4) \\
4, & \text { otherwise }
\end{array}, Q_{4 k+2,1}^{-}=8 P_{2 k+2}, Q_{4 k-1,1}^{-}=4 .\right.
\end{gathered}
$$

Proof All results are seen by using the identities of Theorem 9 and Theorem 10 given in (Koken and Arslan, 2018).

Theorem 11 For the $Q_{n, 1}^{+}$and $Q_{n, 1}^{-}$, the Pell Lucas type recursive relations are valid:

$$
\begin{aligned}
2 Q_{2 k+2,1}^{+}-Q_{2 k, 1}^{+} & =\left\{\begin{array}{ll}
4 q_{k+3}, & \text { if } n \text { is odd } \\
8 P_{k+2}, & \text { otherwise }
\end{array}, \quad Q_{2 k+2,1}^{+}-2 Q_{2 k, 1}^{+}=\left\{\begin{array}{l}
8 P_{k+1}, \text { if } n \text { is odd } \\
-2 Q_{k}, \text { otherwise }
\end{array},\right.\right. \\
2 Q_{2 k+2,1}^{-}-Q_{2 k, 1}^{-} & =\left\{\begin{array}{ll}
8 P_{k+2}, & \text { if } n \text { is odd } \\
2 Q_{k+3}, & \text { otherwise }
\end{array}, \quad Q_{2 k+2,1}^{-}-2 Q_{2 k, 1}^{-}=\left\{\begin{array}{ll}
-4 q_{k}, & \text { if } n \text { is odd } \\
8 P_{k+1}, & \text { otherwise }
\end{array} .\right.\right.
\end{aligned}
$$

Proof By using the definitions of the $Q_{n, 1}^{+}$and $Q_{n, 1}^{-}$, the identities $q_{n+1}+q_{n-1}=4 P_{n}$ and $P_{n-1}+P_{n}=q_{n}$, we obtain all results. 
Corollary 4 When the values of indices vary according to numbers in Lemma 5 and Lemma 6, we can rewrite

$$
\begin{gathered}
Q_{4 k+2,1}^{+}-Q_{4 k, 1}^{+}=2 Q_{2 k+1}, \quad Q_{4 k+2,1}^{-}-Q_{4 k, 1}^{-}=2 Q_{2 k+2}, \quad Q_{4 k, 1}^{+}-Q_{4 k-2,1}^{+}=2 Q_{2 k+1}, Q_{4 k, 1}^{-}-Q_{4 k-2,1}^{-}=2 Q_{2 k}, \\
Q_{4 k+2,1}^{+}-Q_{4 k-2,1}^{+}=4 Q_{2 k+1}, \quad Q_{4 k+2,1}^{-}-Q_{4 k-2,1}^{-}=16 P_{2 k+1} .
\end{gathered}
$$

And also, we have

$$
\begin{array}{ll}
2 Q_{4 k+2,1}^{+}-Q_{4 k, 1}^{+}=8 P_{2 k+2}, & 2 Q_{4 k+2,1}^{-}-Q_{4 k, 1}^{-}=2 Q_{2 k+3}, \\
2 Q_{4 k, 1}^{+}-Q_{4 k-2,1}^{+}=2 Q_{2 k+2}, & 2 Q_{4 k, 1}^{-}-Q_{4 k-2,1}^{-}=8 P_{2 k+1} .
\end{array}
$$

Theorem 12 Some recurrence relation of the $Q_{n, 1}^{+}$and $Q_{n, 1}^{-}$are

$$
\begin{aligned}
& Q_{4 k+4,1}^{+}=4 Q_{4 k+2,1}^{+}-Q_{4 k, 1}^{+}, Q_{4 k+4,1}^{-}=2 Q_{4 k+2,1}^{-}-Q_{4 k, 1}^{-}, \\
& Q_{4 k+2,1}^{+}=2 Q_{4 k, 1}^{+}-Q_{4 k-2,1}^{+}, Q_{4 k+2,1}^{-}=4 Q_{4 k, 1}^{-}-Q_{4 k-2,1}^{-} .
\end{aligned}
$$

Proof By using values of these numbers in Lemma 5, Lemma 6, and the identities $q_{n+1}+q_{n-1}=4 P_{n}$, $P_{n-1}+P_{n+1}=2 q_{n}$, the recurrence relations are obtained.

Corollary 5 There exist some relations between the $Q_{n, 1}^{+}$and $Q_{n, 1}^{-}$numbers;

$$
\begin{array}{ll}
Q_{4 k+4,1}^{+}-Q_{4 k, 1}^{+}=16 P_{2 k+2}=2 Q_{4 k+2,1}^{-}, & Q_{4 k+4,1}^{-}-Q_{4 k, 1}^{-}=4 Q_{2 k+2}=2 Q_{4 k+2,1}^{+}, \\
Q_{4 k+2,1}^{+}-Q_{4 k-2,1}^{+}=4 Q_{2 k+1}=2 Q_{4 k, 1}^{-}, & Q_{4 k+2,1}^{-}-Q_{4 k-2,1}^{-}=16 P_{2 k+1}=2 Q_{4 k, 1}^{+} .
\end{array}
$$

Theorem 13 The Binet formulas for the $Q_{n, 1}^{+}$and $Q_{n, 1}^{-}$are

$$
\begin{gathered}
Q_{4 k, 1}^{+}=2 \sqrt{2}\left(\phi^{2 k+1}-\varphi^{2 k+1}\right), \quad Q_{4 k+2,1}^{+}=2\left(\phi^{2 k+2}+\varphi^{2 k+2}\right), \\
P_{4 k, 1}^{-}=2\left(\phi^{2 k+1}+\varphi^{2 k+1}\right), P_{4 k+2,1}^{-}=2 \sqrt{2}\left(\phi^{2 k+2}-\varphi^{2 k+2}\right) .
\end{gathered}
$$

Proof All results are obtained by using the identities given in (1).

Theorem 14 For Cassini, Catalan and D'ocagne's like identities of the $Q_{4 k, 1}^{+}$and $Q_{4 k, 1}^{-}$, identities in the following are valid;

$$
\begin{gathered}
Q_{4 k+4,1}^{+} Q_{4 k-4,1}^{+}-\left(Q_{4 k, 1}^{+}\right)^{2}=4^{4}, Q_{4(k+r), 1}^{+} Q_{4(k-r), 1}^{+}-\left(Q_{4 k, 1}^{+}\right)^{2}=4^{3} P_{2 r}^{2}, \\
Q_{4 k_{1}+4,1}^{+} Q_{4 k_{2}, 1}^{+}-Q_{4 k_{1}, 1}^{+} Q_{4 k_{2}+4,1}^{+}=2 P_{2\left(k_{1}-k_{2}\right)}, Q_{4 k+4,1}^{-} Q_{4 k-4,1}^{-}-\left(Q_{4 k, 1}^{-}\right)^{2}=-2^{7}, \\
Q_{4(k+r), 1}^{-} Q_{4(k-r), 1}^{-}-\left(Q_{4 k, 1}^{-}\right)^{2}=-2^{5} P_{2 r}^{2}, Q_{4 k_{1}+4,1}^{-} Q_{4 k_{2}, 1}^{-}-Q_{4 k_{1}, 1}^{-} Q_{4 k_{2}+4,1}^{-}=-2^{6} P_{2\left(k_{2}-k_{1}\right)} .
\end{gathered}
$$

Corollary 6 The $\left\{Q_{n, 2}^{+}\right\},\left\{Q_{n, 2}^{-}\right\},\left\{P_{n, 3}^{+}\right\}$and $\left\{P_{n, 3}^{-}\right\}$are constant sequences. 


\section{CONCLUSION}

We examined the altered Pell and Pell Lucas numbers, which are by altering according to two integer parameters of the Pell and Pell Lucas numbers, and obtained the Binet formula, the Cassini, Catalan and D'ocagne's like identities of them. Our results enable us to get outcome in a short time with many interesting problems involving these sequences.

\section{REFERENCES}

Bicknell N, 1975. A primer on the Pell sequence and related sequence, Fibonacci Quart. 13(4), 345349.

Chen KW, 2011. Greatest common divisors in shifted Fibonacci sequences. J. Integer. Seq. 14, 11. 4-7.

Dudley U, Tucker B, 1971. Greatest common divisors in altered Fibonacci sequences. Fibonacci Quarterly, 9: 89-91.

Gül K, 2018. On the $k$ Pell Quaternions and the $k$ Pell Lucas Quaternions. Iğdır University Journal of the Institute of Science and Technology, 8(1): 23-35.

Halıc1 S, Oz S, 2016. On Some Gaussian Pell And Pell Lucas Numbers. Ordu University Journal of Science and Technology, 6(1): 8-18.

Halıc1 S, Oz S, 2018. On Gaussian Pell Polynomials and Their Some Properties. Palestine Journal of Mathematics 7(1), 251-256.
Horadam AF, 1971. Pell identities, Fibonacci Quart. 9(3), 245-263.

Horadam AF, Mahon JM, 1985. Pell and Pell-Lucas Polynomials, Fibonacci Quart. 23(1), 7-20.

Horadam AF, 1994. Applications of Modified Pell Numbers to Representations. Ulam Quart., 3, 34-53.

Karakas M, Karatas A.M, 2017. New Banach sequence spaces that is defined by the aid of Lucas numbers. Iğdır University Journal of the Institute of Science and Technology, 7(4): 103-111.

Koken F, Arslan, S, 2018. GCD Properties of the Altered Pell And Pell Lucas Numbers. IOSR Journal of Mathematics, 14(5): 82-89.

Koshy T, 2001. Fibonacci and Lucas Numbers with Applications. A Wiley-Interscience Publication, Newyork, 672 p.

Koshy T 2014. Pell and Pell Lucas Numbers with Applications. Springer, Berlin, 444 p.

Mahon JM, Horadam AF, 1986. Matrix and Other Summation Techniques for Pell Polynomials. Fibonacci Quart. 24(4), 290-308.

McDaniel WL, 1991. The G.C.D. in Lucas sequences and Lehmer number sequences, Fibonacci Quart. 29, 24-29.

Tasyurdu Y, Cobanoğlu N, Dilmen Z, 2016. On the a New Family of k-Fibonacci Numbers. Erzincan University Journal of Science and Technology, 9(1): 95-101. 